\title{
Ctenophthalmus (Ethioctenophthalmus) KEMmelberg N. SP. (INSECTA : SiphONAPTERA : CTenOPhthalmidAe), PuCE NOUVELLE DE TANZANIE ET DESCRIPTION DE STRUCTURES INTERNES NON SIGNALÉES CHEZ LES MÉCOPTÈroÏDES
}

\author{
LAUDISOIT A.*,** \& BEAUCOURNU J.C.********
}

Summary: CTENOPHTHALMUS (ETHIOCTENOPHTHALMUS) KEMMELBERG N. SP. (InSECTA: Siphonaptera: CTenophthalmidae), a NEW Flea FROM TANZANIA AND DESCRIPTION OF UNKNOWN SMALL STRUCTURES IN THE MECOPTEROIDS

We recently described two new taxa of Siphonaptera

(Ctenophthalmus (Ethioctenophthalmus) teucqae teucqae et C. (E.) teucqae shumeensis Laudisoit \& Beaucournu, 2007) from Lushoto plague focus (West Usambara mountains, Tanzania). A new taxa is here being studied, Ctenophthalmus kemmelberg, an original flea, not only for its genitalia, but also for original structures appearing, and that have not been described yet, at our knowledge, in any Mecopteroid. These are visible in females only. We suggest the name "Teucq's organs" for those remarkable structures.

KEY WORDS : Tanzania, plague focus, Mecopteroidea, Siphonaptera, Ctenophthalmus kemmelberg $\mathrm{n}$. sp.

\section{INTRODUCTION}

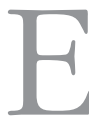
n poursuivant l'étude du foyer pesteux de Lushoto (Tanzanie), des Siphonaptères nouveaux, poten$\checkmark$ tiellement vecteurs au niveau des Rongeurs réservoirs ont été collectés. Nous avons récemment décrit de cette région deux taxa nouveaux (Ctenophthalmus (Ethioctenophthalmus) teucqae teucqae et C. (E.) teucqae shumeensis Laudisoit \& Beaucournu, 2007). Nous étudierons ici un taxon nouveau, Ctenophthalmus kemmelberg, puce originale, non seulement par ses segments génitaux, mais aussi par des structures jusqu'à maintenant non décrites, observables chez les femelles. Nous rappelons (L. \& B., op. cit.) la remarquable richesse faunistique des Monts Usambara, où, inter alia, feu notre Collègue C.A. Hubbard a déjà travaillé avec des résultats remarquables puisqu'il y a décrit, de 1963 à 1967, seize taxa de Siphonaptères.

\footnotetext{
* Université de Liège, Unité de recherches zoogéographiques, Faculté des Sciences, 27, boulevard du rectorat, Bâtiment B22 Botanique, 4000 Liège (Sart-Tilman), Belgique.

** Université d'Anvers, Unité EVOBIO, 171, Groenenborgerlaan, 2020 Antwerpen, Belgique.

**:* Laboratoire de Parasitologie et Zoologie appliquée, Faculté de Médecine, 2, avenue du Professeur Léon Bernard, 35043 Rennes cedex, France.

***** Institut de Parasitologie de l'Ouest, Faculté de Médecine, 2, avenue du Professeur Léon Bernard, 35043 Rennes cedex, France. Correspondance : Jean-Claude Beaucournu.

E-mail : jeanclaude.beaucournu@gmail.com
}

\section{Résumé :}

Nous avons récemment décrit deux taxa nouveaux de Siphonaptères (Ctenophthalmus (Ethioctenophthalmus) teucqae teucqae et C. (E.) teucqae shumeensis Laudisoit \& Beaucournu, 2007) du foyer pesteux de Lushoto (Monts Usambara occidentaux, Tanzanie). Nous étudierons ici un taxon nouveau, Ctenophthalmus kemmelberg, puce originale, non seulement par ses segments génitaux, mais aussi par des structures jusqu'à maintenant décrites, à notre connaissance, chez aucun Mécoptèroïde. Elles sont observables chez les femelles uniquement. Nous proposons d'attribuer à ces structures remarquables le nom d'"organes de Teucq".

MOTS CLÉS : Tanzanie, foyer pesteux, Mécoptèroïdea, Siphonaptères, Ctenophthalmus kemmelberg $n$. sp.

\section{RÉSULTATS}

\section{CTENOPHTHALMUS (ETHIOCTENOPHTHALMUS) KEMMELBERG N. SP.}

\section{- Matériel de description}

Mâle holotype, femelle allotype, un mâle et quatre femelles paratypes sur Grammomys ibeanus (Osgood, 1910), mâle (TE 4248), Magamba, Kwemonho (Tanzanie), lat. 4,75 S, long. 38,28 E, alt. $1642 \mathrm{~m}(+/-15,8 \mathrm{~m})$, 27 juin 2005; trois mâles paratypes, mêmes hôtes, mâles (FZ 832-834), Magamba, Mshelemle, lat. 4,74 S, long. 38,30 E, alt. $1720 \mathrm{~m} \mathrm{(+/-} \mathrm{9,4} \mathrm{m),} 29$ juin 2005; quatre mâles, quatre femelles paratypes, mêmes hôtes, mâles (FZ 904- 906- 907- 908- 910- 913 et 915), mêmes commune et lieu-dit, mêmes coordonnées, 2 juillet 2005; un mâle paratype sur Hylomyscus arcimontensis Carleton et Stanley, 2005, mâle (PZ 01), Gologolo, Grewal (Tanzanie), lat. 4,72 S, long. 38,25 E, alt. $1933 \mathrm{~m} \mathrm{(+/-}$ 9,6 m), 20 juillet 2005 (A. Laudisoit rec.).

Derivatio nominis : le Mont Kemmel (Kemmelberg) est le point culminant d'une région de collines de la province de Flandre occidentale, en Belgique. Son altitude est de $156 \mathrm{~m}$. Ce nom est à la fois celui d'une localité chargée de souvenirs, et celui d'amis pour le premier auteur; mot mis en apposition.

Dépôt des types : holotype, allotype, deux mâles et deux femelles paratypes dans les Collections d'Entomologie du Muséum Royal de l'Afrique centrale de Ter- 
vuren (Belgique); les autres paratypes dans les collections du deuxième auteur, collections ultérieurement déposées au Laboratoire d'Entomologie du Muséum National d'Histoire Naturelle de Paris (France).

\section{- Description}

Smit (1963) place, entre autres, les quatre espèces que nous considérons comme affines de C. (E.) kemmelberg n. sp., à savoir : debrauwerei, devignati, phyris et vanhoofi dans ce qu'il estime être des "ungroupable ethiopian species". Hopkins \& Rothschild (1966), bien que reconnaissant la justesse de vue de Smit, créent pour la majorité (mais non la totalité) des Ctenophthalmus éthiopiens, le sous-genre Ethioctenophthalmus, qu'ils divisent, y compris les "ungroupables species", en plusieurs groupes. Celui qui nous concerne entre dans le groupe "devignati".

Capsule céphalique (figure 1) : cténidie génale composée d'une première dent courte et pointue, légèrement séparée de la deuxième qui est un peu plus longue et surtout plus large ; la base de la troisième empiète sur celle de la deuxième et sa longueur est le double de celle de la première. Rangée frontale supérieure de cinq soies; rangée frontale inférieure de trois soies. Trois rangées occipitales de, respectivement, deux, trois et quatre soies. CEil vestigial, ce qui permet de voir l'arc du tentorium. Palpe labial faisant les 3/4 de la longueur de la coxa I. Area communis présente dans les deux sexes.

Thorax. Prothorax montrant une rangée de six soies par côté ; cténidie de 16 épines, les plus dorsales étant plus longues que le bord libre du segment. Mesothorax : deux pseudo-setae en position dorsale. Metepimeron portant cinq soies; spiracle en pomme de pin. Sulcus de la coxa II, complet.

Abdomen (segments non modifiés) : rangée de soies principale des tergites de six soies, la plus inférieure implantée au-dessous du spiracle. Une spinule sur les segments I-IV, dans les deux sexes, une pouvant exister sur le segment V du mâle. Sternites montrant, respectivement, chez le mâle, 1, 2, 2, 2, 2, 3; chez la femelle, 1, 3, 3, 3, 3, 4 soies. Le sternite II montre, chez la femelle, une microsculpture en empreinte digitale. Trois soies antesensiliales dans les deux sexes, de ratio (en partant de la soie la plus dorsale) $1-3-1,3$, chez le mâle comme chez la femelle.

Abdomen (segments modifiés du mâle) : sternite VIII (figure 2) grand, arrondi postérieurement, ventralement non échancré, portant en situation ventrale huit à dix soies, dont deux grandes submarginales. Segment IX (figure 3) : tergite montrant un manubrium assez large. Basimère : processus basimeris dorsalis (pbd) à apex arrondi portant trois grandes soies; il est légèrement plus haut que le processus basimeris ventralis (pbv), dont il est séparé par une encoche petite, mais nette. Le pbv montre deux parties, séparées par une petite "entaille" plus ou moins accusée : la plus dorsale est tabulaire et porte une petite soie; la plus ventrale est oblique avec deux grandes soies, dites acétabulaires. Télomère (processus telomeris) plus haut que large (rapport $\mathrm{h} / \mathrm{l}=2 / 1)$; son apex est quadrangulaire avec cinq sensillae (quatre chez un paratype). Fovea peu distincte : elle semble proche du bord antérieur et de forme allongée. Le bord postérieur est d'abord largement concave, puis convexe, l'apex de la convexité portant la tétrade de soies classique. On note une discrète crénulation de sa partie postéro-ventrale. Sternite relativement court et à segments épais; l'apex du bras distal est arrondi obliquement et montre une douzaine de soies dont la moitié sont longues.

Phallosome (figure 4) caractéristique. Lamina media large et relativement courte dans sa partie libre; sclérite de Ford, en "U" renversé; tubus interior mal discernable en fonction des divers organites qui se superposent (nous l'avons, volontairement, rendu plus visible sur notre dessin); hamulus à contours arrondis. Le point le plus notable est l'extrême développement du lobe medio-dorsal qui évoque, par sa taille seulement, celui de $C$. devignati. Parmi les espèces décrites à ce jour, le phallosome, à lui seul, permettra d'identifier C. kemmelberg n. sp.

Abdomen (segments modifiés de la femelle) (figure 5) : sternite VII à marge distale trilobée, le lobe dorsal n'étant qu'une brusque courbure de la moitié dorsale de la marge distale. Il porte en position ventrale cinq courtes soies et quatre longues et épaisses. Tergite VIII : deux soies au-dessus du spiracle qui est de forme classique en "Y". Sétation regroupée à la partie ventrale : trois longues et fortes soies submarginales et six ou sept, plus courtes et plus antérieures. Une large zone verticale, légèrement pigmentée, allant du spiracle à la deuxième angulation du tergite VIII, correspond au segment IX; nous ne pouvons isoler le tergite du sternite. À la même hauteur que le sensilium, deux formations internes, l'une à gauche, l'autre à droite de l'abdomen, sont visibles; nous y reviendrons. Sternite VIII, en triangle très allongé, pigmenté. Le segment XI ou proctiger (figure 5) n'est représenté, ici, que pour mettre en évidence la situation des formations que nous venons d'évoquer.

Spermathèque, ducti (figure 6) : spermathèque plutôt grande; la hilla est courbée à angle droit et est aussi longue que la bulga; celle-ci est rétrécie dorso-postérieurement, évoquant celle de $C$. debrawwerei. Le vagin est de structure complexe comme chez les espèces apparentées. Ductus bursae sub-rectiligne; perula en cœur ou en corolle : le départ des deux ducti est visible, le ductus spermathecae étant au-dessus du ductus obturatus. Si nous nous fions à ce que nous observons chez de rares femelles de $C$. debrauwerei où ces ducti sont visibles, ces deux conduits sont de même longueur. Rappelons que Smit (1963) écrivait “...in van- 

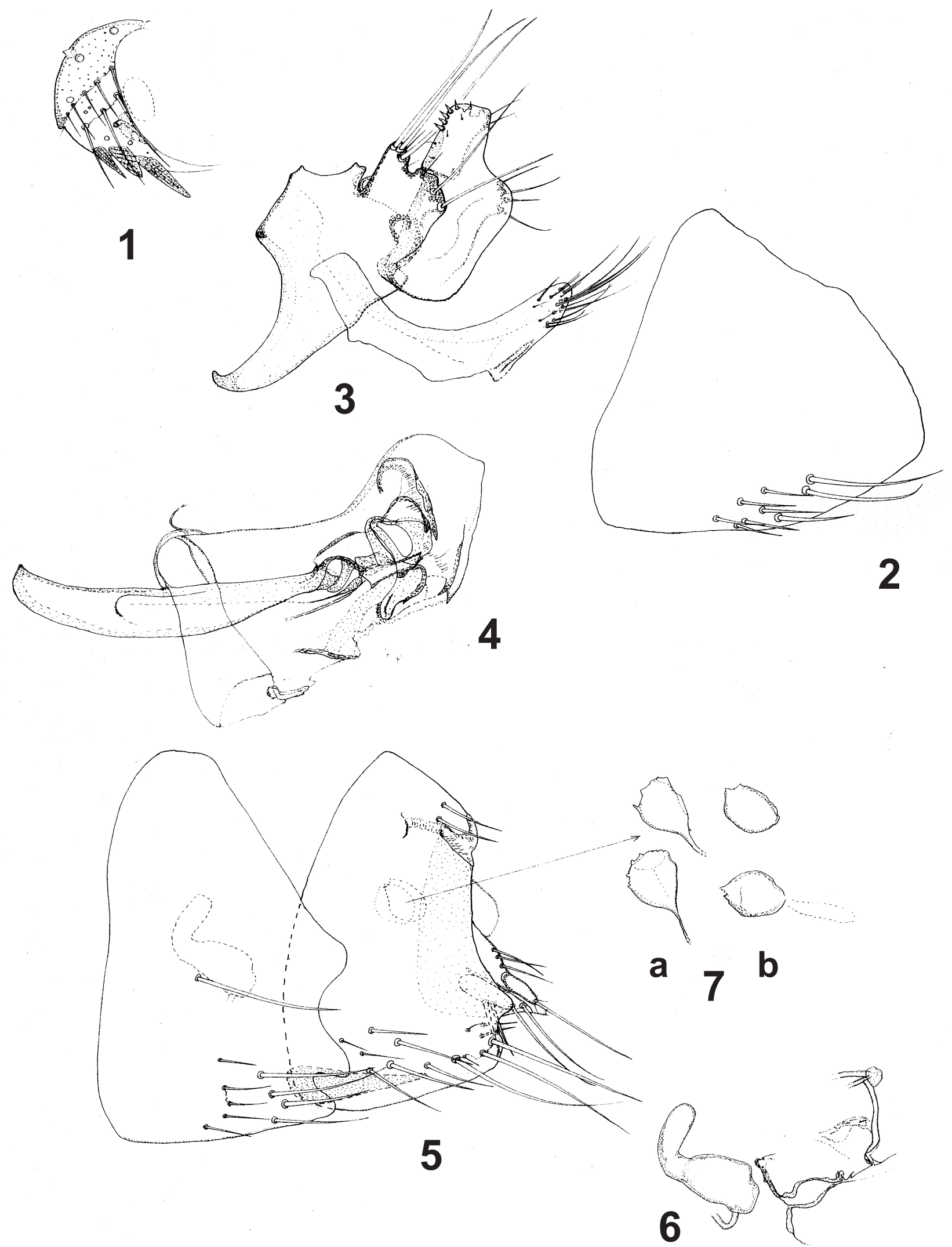

Figs 1-7. - Ctenophthalmus (E.) kemmelberg n. sp. 1 : capsule céphalique du mâle holotype. 2 : sternite VIII de l'holotype. 3 : segment IX de l'holotype. 4 : phallosome d'un paratype. 5 : sternite VII, segment VIII, segment IX, proctiger, organes de Teucq de l'allotype. $6:$ spermathèque et ducti (dessin composite, allotype et un paratype. 7 : organes de Teucq de l'allotype (7a) et d'un paratype (7b) (le dessin du bas représente l'organe situé du coté gauche, celui du haut représente l'organe situé à droite; ils sont artificiellement décalés). 
boofi and debrauwerei only the ductus spermathecae could be seen (Fig. 212)".

\section{. Formations internes (figure 7)}

Ces "formations", que nous avons ci-dessus situées anatomiquement, semblent plus ou moins lenticulaires et sont nettement séparées l'une de l'autre. Leur surface est membraneuse, irrégulière, montrant quelques "aspérités" à leur surface. La majorité montre un prolongement vers le bas et, plus ou moins, vers l'extrémité anale du proctodeum, sans que nous puissions affirmer une continuité avec le tube digestif. Précisons tout de suite qu'il ne s'agit ni d'artefacts, ni de formations parasitaires. La preuve en est apportée par leur présence, chaque fois en double, chez nos quatre femelles; elles sont toujours au même emplacement dans l'abdomen : l'une dans la partie gauche (le côté le plus proche de l'observateur chez une puce montée correctement : capsule céphalique à gauche et sternites vers le bas), l'autre à droite. Chez l'insecte, elles se superposent pratiquement (figure 5) : c'est artificiellement que nous les avons décalées sur les deux autres figures (figure 7 , a-b). Il ne s'agit pas d'excroissances rectales ("rectal pads", Rothschild et al., 1986) de l'ampoule rectale : les six présentes dans ce genre sont toutes visibles dans notre matériel. Il ne peut s'agir des glandes salivaires, qui sont également au nombre de deux : elles sont beaucoup plus antérieures, et plus ventrales, au niveau des premiers segments abdominaux, chez les Siphonaptères; si chez Phacopsylla inexpectata mâle, elles sont en position médiane par rapport au corps de l'insecte, cela est du à la constriction extrême de l'abdomen (Beaucournu et al., 1996). Nous devons écarter les tubules de Malpighi, qui sont des structures tubulaires, ce qui n'est manifestement pas le cas ici; pour la même raison, nous ne pouvons évoquer des sections trachéales qui, de plus, montrent une sclérification interne nette ( $c f$. Beaucournu et al., 1999), ni même, mais cette fois non sclérifiées, des ovarioles. Enfin, tout rattachement à des spermathèques, ou à des $d u c t i$, serait en opposition totale avec les nombreux cas observés (Beaucournu et al., 1988). À notre connaissance, aucun Mécoptéroïde ne semble présenter d'organes analogues. Nous proposons, pour éviter des périphrases fastidieuses, de nommer ces formations les "organes de Teucq".

En résumé, il nous est malheureusement impossible de donner l'origine, et surtout la fonction de ces organes; celle-ci existe certainement, aucune formation n'étant "gratuite" dans le monde vivant. Ceci est d'autant plus curieux que Ctenophthalmus Kolenati, le genre ici concerné, est riche d'environ 170 espèces : aucune des dizaines que nous avons étudiées, souvent en très grand nombre, ne montre ces formations. Il n'est pas rare chez les Siphonaptères (comme chez beaucoup d'autres arthropodes) de noter chez une ou quelques espèces d'un genre ou d'une famille donné, un organe "insolite". Par exemple, la glande de Wagner est présente chez de nombreux Ceratophyllidae (Ceratophyllinae), mais dans le genre Macrostylophora Ewing, certaines espèces la possèdent, d'autres, a priori voisines, n'en ont pas. On peut citer, de façon encore plus représentative, l'organe de Smit, organe présumé sensoriel présent seulement chez la femelle de Rhadinopsylla (Actenophthalmus) isacantha (Rothschild, 1907) (Ctenophthalmidae), alors que ce genre est riche de plus de 60 espèces.

\section{- Dimensions}

Mâles variant de 1,8 à 2,1 mm (holotype : 1,9 mm); femelles variant de 2,4 à 2,7 mm (allotype : 2,7 mm).

\section{COMMENTAIRES}

- 'individualité de Ctenophthalmus (Ethio.) kemmelberg n. sp. n'est pas discutable étant donné $\checkmark$ la morphologie des genitalia du mâle, et en particulier celle du phallosome. Il faut toutefois reconnaitre que C. (E.) nyikensis Smit, 1962 (groupe "eumeces"), décrite du Malawi, et dont la femelle est inconnue à ce jour, en est proche par la morphologie de son segment IX, bien que le pbv soit un peu différent et que la tétrade de soies du télomère soit insérée nettement plus bas; si les femelles de C. (E.) kemmelberg sont moins originales et pourraient faire évoquer celles de C. (E.) debrauwerei, la présence chez elles, et elles seules pour le moment, des organes de Teucq ne permet aucune confusion.

Grammomys ibeanus est connu depuis longtemps, mais Hylomyscus arcimontensis a été isolé récemment (2005) du complexe "denniae" et est de dispersion relativement réduite; il est cantonné aux forêts humides, entre 900 et plus de 2400 m d'altitude. C'est également le biotope des G. ibeanus parasités. H. arcimontensis est actuellement signalé du nord du Malawi à l'est de la Tanzanie, suivant une bande s'étirant du sud-ouest à l'est, en fonction des critères écologiques de ce rongeur (Carleton \& Stanley, 2005). Il est curieux de noter que ces auteurs rappellent que "In the EAM (East African Montains) archipelago, $\mathrm{H}$. arcimontensis is the most abundant arboreal small rodent in montane forest, sharing this habitat with Dendromus mesomelas, Beamys hindei, Grammomys ibeanus, G. macmillani, and Graphiururus murinus". Or, que l'hôte primaire soit G. ibeanus, ce qui semble s'imposer, ou $H$. arcimontensis, tous les deux sont des rongeurs arboricoles, ce qui ne correspond pas à la quasi-cécité du parasite ( $c f$. supra). Décrivant de Tanzanie, Xenopsylla microphthalma capturée sur Beamys hindei, Beaucournu \& Kock (1996), ont eu le même problème, et ces auteurs pensaient que $B$. hindei, n'était sans doute 
pas l'hôte primaire; mais ils citaient également Hubbard (1970) écrivant "qu'il avait eu l'impression qu'il est difficile à $B$. hindei de grimper et qu'il tombe facilement". Il ne nous appartient pas de résoudre cette discordance.

\section{REMERCIEMENTS}

$\mathrm{P}$ our leur précieuse aide sur le terrain comme dans la vie au quotidien dans les montagnes Usambara, nous remercions Michael Mkande, Joseph C. Hizza, les villageois du district de Lushoto, et le père Joseph Shio CSSP de la Mission des Spiritains qui nous ont chaleureusement hébergés et accompagnés durant nos séjours. Pour le support logistique, matériel, ou financier, nous sommes reconnaissants envers le FRIA, le VLIR, les Professeurs B.S. Kilonzo, R.H. Makundi, R. Machang'u, et toute l'équipe de SUA Pest Management Center (Sokoine University of Agriculture), l'Université d'Anvers et en particulier le Professeur H. Leirs, Madame M.D. Simonet, Ministre wallone de la recherche scientifique et de l'éducation et le Lion's Club de Liège. Enfin, nous dédions cette description aux hôtes du "Kemmelberg", source d'inspiration anversoise.

\section{RÉFÉRENCES}

Beaucournu J.C., Gomez M.S. \& Ménier K. Apports de la tératologie à l'étude des Siphonaptères : discussion à propos de trois cas de stigmates surnuméraires. Boletin de la Asociacion espanola de Entomologia, 1999, 23, 249-256.

BeAucournu J.C. \& Kock D. Xenopsylla microphthalma n. sp., puce nouvelle de Tanzanie (Insecta : Siphonaptera : Pulicidae). Senckenbergiana biologica, 1996, 75, 159-162.

Beaucournu J.C., Launay H. \& Sklair A. Les anomalies des spermathèques et des conduits génitaux chez les Siphonaptères (Insecta). Revue bibliographique et cas personnels. Annales de Parasitologie humaine et comparée, 1988, 63, 64-75.

Beaucournu J.C., Mahamat Tahir T., Branquet F. \& Horak I.G. Données complémentaires sur la morphologie, l'anatomie et les possibles interactions hôte-parasite de Phacopsylla inexpectata (Smit, 1950) (Insecta : Siphonaptera : Pulicidé). Parasite, 1996, 3, 69-75.

Carleton M.D. \& Stanley W.T. Rewiew of the Hylomyscus denniae complex (Rodentia: Muridae) in Tanzania, with a description of a new species. Proceedings of the Biological Society of Washington, 2005, 118, 619-646.

Hopkins G.H.E. \& RothschiLD M. An illustrated catalogue of the Rothschild collection of fleas (Siphonaptera) in the British Museum (Natural History) - Vol. IV: Hystrichopsyllidae. The British Museum, London, 1966, 549 p., 12 pl.

Hubbard C.A. A first record of Beamys from Tanzania, with observations of its breeding and habits in captivity. Zoologia africana, Cape Town, 1970, 5, 229-236.
Laudisoit A. \& Beaucournu J.C. Deux nouveaux Ctenophthalmus (Insecta : Siphonaptera : Ctenophthalmidae) de Tanzanie. Parasite, 2007, 14, 101-105.

Rothschild M., Schlein Y. \& ITo S. A colour atlas of insect tissues, via the flea. Wolfe Publishing Ltd., London, 1986, 184 p., très nombreuses figures.

Smit F.G.A.M. Species-groups in Ctenophthalmus (Siphonaptera: Hystrichopsyllidae). Bulletin of the British Museum (Natural History), Entomology, London, 1963, 14, 107-152, $58 \mathrm{pl}$.

Reçu le 20 mars 2007 Accepté le $1^{\text {er }}$ juin 2007 
ARASITE, Journal de la Société Française de Parasitologie, publie, en anglais et en français, des mémoires originaux,

des notes de recherche, des informations (lettres à la rédaction, analyse d'ouvrages), des actes de congrès ainsi que des mises au point sollicitées sur des sujets spécifiques. Tous les aspects de la parasitologie sont abordés, qu'il s'agisse de recherche fondamentale ou appliquée dans des domaines aussi divers que l'entomologie, l'helminthologie, la protozoologie, l'épidémiologie, l'écologie, la thérapeutique, l'immunologie, la biologie moléculaire, la génétique...

ARASITE est une publication internationale. Les manuscrits venant de chercheurs de tous pays sont les bienvenus, qu'ils soient ou non membres de la Société française de Parasitologie. L'auteur soumettant un manuscrit garantit son caractère original et qu'il n'a pas été soumis ailleurs pour publication. Il s'engage à demander l'autorisation de l'éditeur pour toute reproduction, même partielle, de son article dans une autre publication. L'auteur principal doit confirmer par écrit l'accord de tous les co-signataires pour la publication de leur manuscrit dans PARASITE.

Tout manuscrit doit être adressé en trois exemplaires avec la version informatique correspondante (texte, figures et tableaux sur fichiers séparés) au : Dr Jean-Marc Dumas, 64 avenue du Général de Gaulle, F-92130 Issy-les-Moulineaux. Il sera enregistré sous un numéro de référence et soumis à l'appréciation anonyme de deux lecteurs, avant acceptation par le Comité de Rédaction.

Les Mémoires originaux seront rédigés en français ou en anglais, et comporteront un maximum de 25 pages de format A4, illustrations comprises, dactylographiées avec un double interlignage à raison de 25 lignes par page et 60 signes par ligne. La terminologie à adopter est celle du Code International de Nomenclature.

Chaque manuscrit doit être présenté ainsi :

a. Une page de titre mentionnant le titre de l'article (pas plus de 150 signes), les nom et initiales des prénoms des auteurs (limités à 6), les nom et adresse de l'établissement dans laquelle le travail a été effectué, une liste de mots-clés en français et en anglais ; les numéros de téléphone, de télécopie et d'e-mail de l'auteur chargé de la correspondance seront indiqués. Un titre courant de 50 signes au plus sera fourni.

b. Afin de préserver l'anonymat des auteurs, la première page de texte devra comporter un rappel du titre. Un résumé de 8 à 15 lignes sera ajouté, rédigé en français, en anglais et le cas échéant dans la langue de l'auteur, accompagné de la traduction du titre.

c. Veuillez découper le texte en Introduction - Matériel et méthodes (les informations doivent être suffisantes pou permettre à d'autres expérimentateurs de répéter l'expérience) - Résultats - Discussion - Remerciements Références.

Seules les références appelées dans le texte (nom de l'auteur, année) et acceptées pour publication doivent figurer à la fin du texte en un index alphabétique, conformément aux exemples suivants :

de Champs C., Pelloux H., Dechelotte P., Giraud J.C., Bally N. \& Ambroise-Thomas P. Toxoplasma gondii infection in rats by the RH strain: inoculum and age effects. Parasite, 1998, 5, 215-218.

Nakornchai S. \& Anantavara S. Oxygen free radicals in malaria, in : Lipid-Soluble Antioxidants, Biochemistry and Clinical Applications. Ong A.S.H. \& Packer L. (eds), Birkhauser Verlag, Basel, 1993, $355-362$.

d. Nomenclature : les auteurs doivent suivre le Code international de nomenclature en zoologie (ICZN, 1999, 4ème ed.). Quand un taxon est cité pour la première fois dans le texte, il doit être suivi du nom de son auteur et de la date de création. La bibliographie ne doit mentionner que les références citées dans le texte. Cependant, pour les travaux de systématique, les auteurs peuvent, s'ils le souhaitent, ajouter les références qui concernent les auteurs de taxa.

e. Les figures et photos en noir et blanc seront remises avec leurs légendes en même temps que le texte, chaque figure étant accompagnée d'une échelle en microns ou en millimètres, de l'indication de son emplacement dans le texte et de la réduction éventuellement désirée par l'auteur. D'éventuelles planches en quadrichromie seront à la charge de l'auteur.

Protocoles thérapeutiques et expérimentations animales doivent être conduits selon les règles en vigueur, sous la seule responsabilité des auteurs.

Les Mises au point sollicitées, de 35 pages dactylographiées au plus, et les Notes de recherche, limitées à 6 pages dactylographiées et à 250 mots pour chaque résumé, obéissent globalement aux mêmes recommandations.

Les Lettres à la rédaction seront de 2 pages dactylographiées au maximum et seront publiées après accord du Comité de Rédaction. Certains cas cliniques seront acceptés dans cette rubrique.

\section{Frais techniques}

- Pour chaque article accepté, il sera demandé aux auteurs la somme de 120 euros de frais de publication. L'auteur correspondant recevra en retour 25 tirés à part et la version électronique .pdf de l'article. Toute demande d'exemplaires supplémentaires sera effectuée lors de la relecture des épreuves et accompagnée du règlement correspondant.

- Au-delà de 8 pages, chaque page de mémoire imprimée sera facturée 100 euros aux auteurs.

- Le coût lié aux reproductions en quadrichromie est à la charge des auteurs. 